\title{
Article \\ FOXE1 Gene Dosage Affects Thyroid Cancer Histology and Differentiation In Vivo
}

\author{
Sara C. Credendino ${ }^{1,2}$, Carmen Moccia ${ }^{1}{ }^{(0}$, Elena Amendola ${ }^{2}{ }^{(}$, Giuliana $^{\prime}$ Avino $^{1}$, Luigi Di Guida ${ }^{1}$, \\ Eduardo Clery $^{3}$, Adelaide Greco ${ }^{4,5}$, Claudio Bellevicine ${ }^{3}{ }^{-}$, Arturo Brunetti ${ }^{4}$, Mario De Felice ${ }^{1,2}(\mathbb{D}$ \\ and Gabriella De Vita ${ }^{1, *(1)}$
}

1 Department of Molecular Medicine and Medical Biotechnology, University of Naples Federico II, via Pansini 5, 80131 Naples, Italy; saracarmela.credendino@unina.it (S.C.C.); Carmen.moccia26@gmail.com (C.M.); giul.davino@gmail.com (G.D.); luigi.diguida@unina.it (L.D.G.); mario.defelice@unina.it (M.D.F.)

2 Institute of Experimental Endocrinology and Oncology "G. Salvatore", National Research Council (CNR), via Pansini, 5, 80131 Naples, Italy; elena.amendola@unina.it

3 Department of Public Health, University of Naples Federico II, via Pansini 5, 80131 Naples, Italy; eduardoclery87@gmail.com (E.C.); claudio.bellevicine@unina.it (C.B.)

4 Department of Advanced Biomedical Sciences, University of Naples Federico II, via Pansini 5, 80131 Naples, Italy; adelaide.greco@unina.it (A.G.); arturo.brunetti@unina.it (A.B.)

5 CEINGE Advanced Biotechnology S.C.AR.L., via Gaetano Salvatore 486, 80145 Naples, Italy

* Correspondence: gdevita@unina.it; Tel.: +39-081-746-3240

Citation: Credendino, S.C.; Moccia, C.; Amendola, E.; D'Avino, G.; Di Guida,

L.; Clery, E.; Greco, A.; Bellevicine,

C.; Brunetti, A.; De Felice, M.; De Vita, G. et al. FOXE1 Gene Dosage Affects Thyroid Cancer Histology and Differentiation in vivo. Int. J. Mol. Sci. 2021, 22, 25. https://dx.doi.org/10.3390/ ijms22010025

Received: 30 November 2020 Accepted: 16 December 2020 Published: 22 December 2020

Publisher's Note: MDPI stays neutral with regard to jurisdictional claims in published maps and institutional affiliations.

Copyright: (c) 2020 by the authors. Licensee MDPI, Basel, Switzerland. This article is an open access article distributed under the terms and conditions of the Creative Commons Attribution (CC BY) license (https: / / creativecommons.org/ licenses/by/4.0/).

\begin{abstract}
The transcription factor Forkhead box E1 (FOXE1) is a key player in thyroid development and function and has been identified by genome-wide association studies as a susceptibility gene for papillary thyroid cancer. Several cancer-associated polymorphisms fall into gene regulatory regions and are likely to affect FOXE1 expression levels. However, the possibility that changes in FOXE1 expression modulate thyroid cancer development has not been investigated. Here, we describe the effects of FOXE1 gene dosage reduction on cancer phenotype in vivo. Mice heterozygous for FOXE1 null allele (FOXE1 ${ }^{+/-}$) were crossed with a $B R A F^{\mathrm{V} 600 \mathrm{E}}$-inducible cancer model to develop thyroid cancer in either a FOXE1 $1^{+/+}$or $F O X E 1^{+/-}$genetic background. In $F O X E 1^{+/+}$mice, cancer histological features are quite similar to that of human high-grade papillary thyroid carcinomas, while cancers developed with reduced FOXE1 gene dosage maintain morphological features resembling less malignant thyroid cancers, showing reduced proliferation index and increased apoptosis as well. Such cancers, however, appear severely undifferentiated, indicating that FOXE1 levels affect thyroid differentiation during neoplastic transformation. These results show that FOXE1 dosage exerts pleiotropic effects on thyroid cancer phenotype by affecting histology and regulating key markers of tumor differentiation and progression, thus suggesting the possibility that FOXE1 could behave as lineage-specific oncogene in follicular cell-derived thyroid cancer.
\end{abstract}

Keywords: FOXE1; thyroid cancer susceptibility; mouse model; differentiation; proliferation

\section{Introduction}

Thyroid cancer is one of the most common endocrine malignancies [1]. Papillary thyroid carcinoma (PTC) is the most frequent among the different thyroid cancer histotypes. It originates from thyroid follicular cells and accounts for $80 \%$ of all thyroid neoplasia cases [2,3]. Notably, among cancers with no Mendelian inheritance, PTC shows the highest relative risk in the first-degree relatives of probands. Despite this evidence indicating that PTC has a strong genetic component, the genes involved in PTC predisposition are poorly characterized [4]. Recently, FOXE1, a transcription factor expressed in the thyroid since early developmental stages and throughout adult life, has been associated with thyroid cancer susceptibility [5,6]. The A allele of single nucleotide polymorphism (SNP) rs965513 close to the FOXE1 genomic locus is highly associated with increased PTC risk [7]. This is a lead SNP falling within a linkage disequilibrium block [chromosome 
9: 100486812-100566532 (hg19)] that contains other co-inherited SNPs acting, in turn, as functional variants of three long-range FOXE1 enhancers, affecting FOXE1 expression [8]. Several other polymorphisms along the FOXE1 gene, or in its genomic region, have been associated with both increased susceptibility to PTC and more aggressive tumors in different case-control studies, including an investigation on Chernobyl accident-derived thyroid carcinoma [9-11]. In particular, the rs1867277 SNP, located in the FOXE1 promoter region, has been positively associated with the severity of histopathological features and lymph node invasion [10,12]. Landa et al. demonstrated that the two alleles of SNP rs1867277 regulate the FOXE1 transcription rate by differentially recruiting USF1/USF2 transcription factors [12]. Beyond genomic variants, different FOXE1 expression levels have been associated with several clinical and pathological cancer parameters, such as extracapsular invasion, lymph node metastasis, and staging [13]. Recently, FOXE1 expression levels have been positively associated with thyroid cancer differentiation degree, however the same study shows that silencing of FOXE1 in vitro decreases migratory and the invasive ability of thyroid cells [14]. Overall, the landscape emerging from the literature strongly indicates that either due to genetic variants of regulatory elements or to still unidentified trans-acting factors, the expression level of Foxe1 is the likely determinant underlying different susceptibility to PTC and/or different cancer phenotypes. Despite these evidences, the demonstration of a cause-effect relationship between FOXE1 expression levels and thyroid cancer phenotype in vivo is still missing.

Here, we investigate if FOXE1 gene dosage is able to influence the outcome of experimental thyroid carcinoma in vivo by using a $B R A F$ oncogene-induced thyroid cancer model with heterozygous Foxe1 knockout allele. We show that genetically reduced Foxe1 levels impact on tumor histology, proliferation, and differentiation. Our data indicate that the abundance of this transcription factor is relevant for determining thyroid cancer features, thus suggesting that FOXE1 could play a dual role in thyroid follicular cells as it is absolutely required for normal gland development, while also being able to affect several aspects of thyroid cancer phenotype, possibly acting as a lineage-specific oncogene.

\section{Results}

\subsection{Generation of FOXE1 Heterozygous Knockout Mouse Model of Thyroid Cancer}

To investigate the role of FOXE1 gene dosage in thyroid cancer development, we used the double transgenic Tg-rtTA/TetOBRAF ${ }^{\mathrm{V} 600 \mathrm{E}}$ mouse model (BRAF) bearing the reverse tetracycline transactivator (rtTA) under the control of the thyroglobulin $(\mathrm{Tg})$ promoter and the $B R A F^{\mathrm{V} 600 \mathrm{E}}$ oncogene under the control of a modified tetracycline operator (TetO). Doxycycline (Dox) induces the expression of $B R A F^{\mathrm{V} 600 \mathrm{E}}$ (Figure $1 \mathrm{~A}$ ), leading to the rapid development of thyroid tumors resembling high-grade PTC [15]. Reduction of FOXE1 expression levels was obtained by crossing BRAF mice with mice heterozygous for FOXE1 null allele $\left(F O X E 1^{+/-}\right)$as homozygous FOXE1 ${ }^{-/-}$are not viable [16]. The crossing generates a total of eight different genotypes, as summarized in Figure 1B. Further analyses were

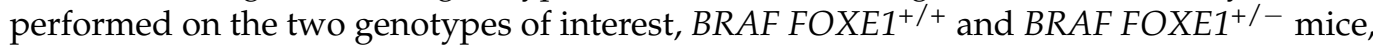
both double transgenic and with bi- or mono-allelic FOXE1 expression, respectively, and $F O X E 1^{+/+}(\mathrm{WT})$ and $F O X E 1^{+/-}$as controls. Representative genotype analysis of selected mice is shown in Figure 1C. Reduction of FOXE1 gene expression in FOXE1 ${ }^{+/-}$litters was

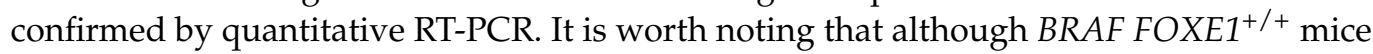
show a reduction (not statistically significant) in FOXE1 expression with respect to WT, FOXE1 levels are lower in each FOXE1 $1^{+/-}$mouse line with respect to the corresponding $\mathrm{FOXE1}^{+/+}$control line (Figure 1D). In the absence of Dox treatment, thyroids belonging to mice of different genotypes are indistinguishable by Hematoxylin and Eosin (H\&E) staining (Figure S1). 
A

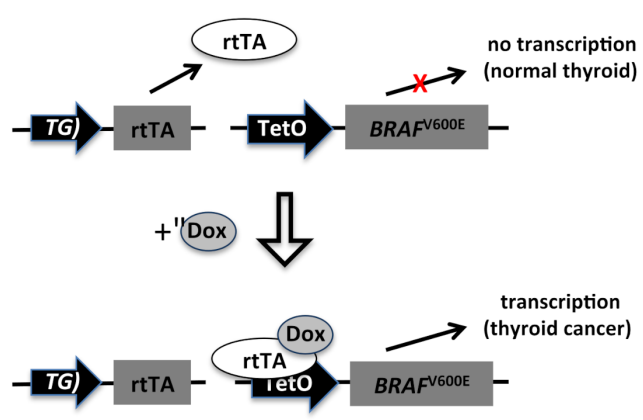

C

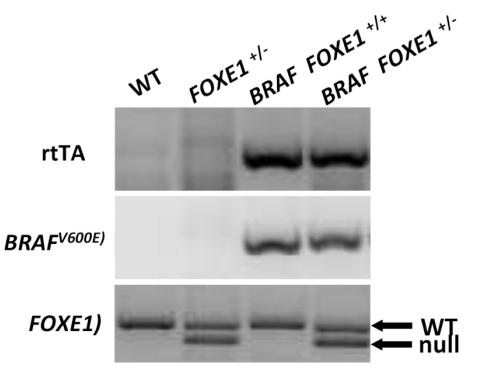

B

\begin{tabular}{|c|c|c|c|}
\hline \multirow[t]{2}{*}{$x$} & \multirow[b]{2}{*}{ gametes } & \multicolumn{2}{|c|}{ FOXE1 +/- } \\
\hline & & FOXE1+" & FOXE1\#' \\
\hline \multirow{4}{*}{$\begin{array}{l}\text { Tg-rtTA } \\
\text { TetO- } \\
\text { BRAF } \\
\text { FOXE1+/+ }\end{array}$} & FOXE1+" & FOXE1 ${ }^{+/+}(\mathrm{WT})$ & FOXE1/- \\
\hline & $\begin{array}{l}\text { TetO\#BRAF } \\
\text { FOXE1+" }\end{array}$ & $\begin{array}{l}\text { TetO-BRAF } \\
\text { FOXE0 } 1^{+/+}\end{array}$ & $\begin{array}{l}\text { TetO-BRAF } \\
\text { FOXE00E }^{+/-}\end{array}$ \\
\hline & $\begin{array}{l}\text { Tg\#ttTA" } \\
\text { FOXE1+" }\end{array}$ & $\begin{array}{l}\text { Tg-rtTA } \\
\text { FOXE1 }^{+/+}\end{array}$ & $\begin{array}{l}\text { Tg-rtTA } \\
\text { FOXE1 }^{+/}\end{array}$ \\
\hline & $\begin{array}{l}\text { Tg\#tTA" } \\
\text { TetO\#BRAF }{ }^{\text {V600E" }} \\
\text { FOXE1+" }\end{array}$ & $\begin{array}{l}\text { Tg-rtTA } \\
\text { TetO-BRAF } \\
\text { FOXEOE }^{+/+} \\
\left(\text {BRAF FOXE1 }^{+/+} \text {) }\right.\end{array}$ & $\begin{array}{l}\text { Tg-rtTA } \\
\text { TetO-BRAF } \\
\text { FOXE1 }^{+/-} \\
\text {(BRAF FOXE1/-) }\end{array}$ \\
\hline
\end{tabular}

D

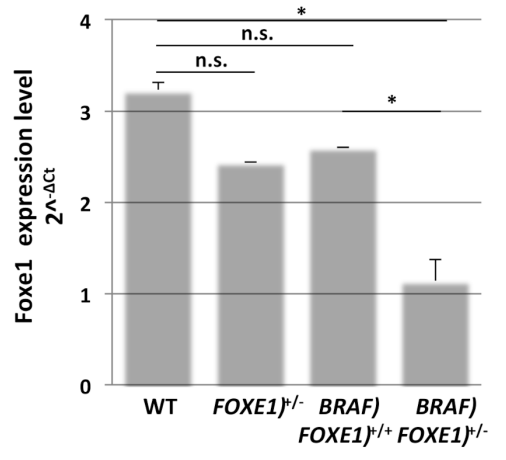

Figure 1. FOXE1 ${ }^{+/-}$mouse model of thyroid cancer. (A). Schematic representation of the Tg-rtTA/TetOBRAF ${ }^{\mathrm{V} 600 \mathrm{E}}$ double transgenic inducible mouse model of thyroid cancer $(B R A F)$. The thyroglobulin $(\mathrm{Tg})$ promoter drives the thyroid-specific expression of the reverse tetracycline transactivator (rtTA). In the presence of doxycycline (Dox), the rtTA is able to induce expression of a BRAF oncogene $\left(B R A F^{\mathrm{V} 600 \mathrm{E}}\right)$ under the control of the Tet operator (TetO) and thyroid cancer develops. (B). Double transgenic BRAF mice were crossed with FOXE1 heterozygous knock-out mice $\left(F O X E 1^{+/-}\right)$to obtain $B R A F$ mice in both $F O X E 1^{+/+}$and $F O X E 1^{+/-}$genetic background. Punnet Square of the cross is shown, with genotypes used in the study shaded in gray. (C). Offspring were genotyped by PCR for the two transgenes rtTA and BRAF and for FOXE1 wild type

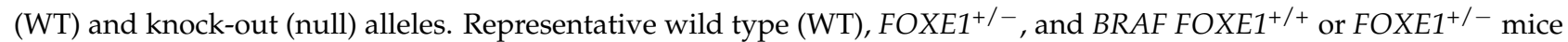
genotypes are shown. (D). FOXE1 levels were evaluated by quantitative RT-PCR analysis on total RNA extracted from

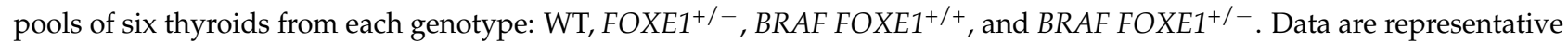
of three different experiments and are reported as actin-normalized $\left(2^{\wedge}-\Delta C_{t}\right)$ values. Means $\pm S D$ are shown. ${ }^{*} p<0.05$, n.s. not significant.

\subsection{Thyroid Cancer Histology in BRAF FOXE1 ${ }^{+/-}$Mice}

Mice were divided into experimental groups, depending on the genotype and the treatment they underwent, each consisting of six animals (Figure S2A). Dox treatments were performed for one week as described in methods on WT, FOXE1 ${ }^{+/-}, B R A F F O X E 1^{+/+}$, and $B R A F F O X E 1^{+/-}$, then thyroids were dissected and analyzed by H\&E staining. As expected, normal thyroid histology in both WT and $F O X E 1^{+/-}$control mice was not affected

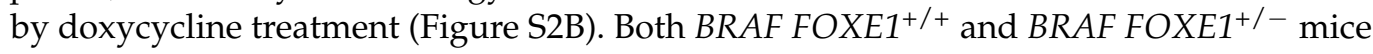
instead developed thyroid cancers, which show different morphologies. Specifically, $B R A F$ $F O X E 1^{+/+}$thyroid tissue displays a dramatic loss of the normal follicular structure, featuring a solid growth pattern as expected for this cancer model [15], while BRAF FOXE1 ${ }^{+/-}$ glands show empty structures, resembling residual follicle lumens (Figure 2A). Follicle-like structures were counted in seven fields for each of the six analyzed thyroids for each genotype, confirming that such structures are significantly more represented in thyroid cancers of $B R A F F O X E 1^{+/-}$mice with respect to that of $B R A F F O X E 1^{+/+}$mice (Figure $2 \mathrm{~B}$ ). 
A

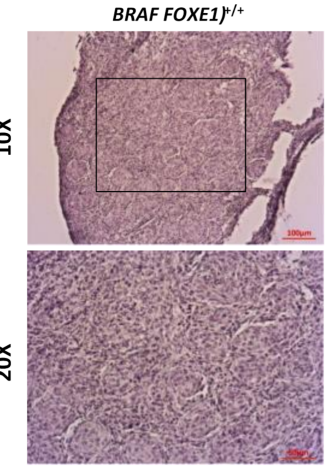

BRAF)FOXE1 +/

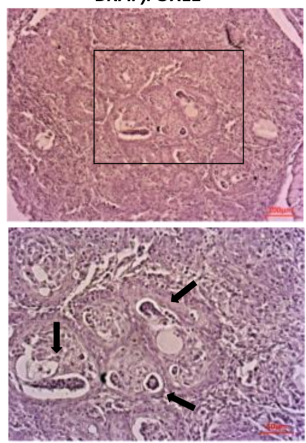

B

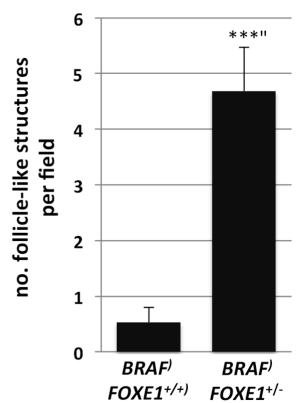

Figure 2. Thyroid cancer in FOXE1 $1^{+/-}$mice shows follicle-like structures. $B R A F F O X E 1^{+/+}$and $B R A F$ $F O X E 1^{+/-}$mice were treated with doxycycline for one week to induce thyroid cancer. (A). Hematoxylin and eosin staining was performed on $7 \mu \mathrm{m}$ sections. Here, $10 \times$ (upper panel) and $20 \times$ of the boxed area (lower panel) magnifications are shown. Black arrows indicate residual follicle-like structures. Each image is representative of six different images. (B). Follicle-like structures were manually counted in 7 fields per mouse $\left(n=6\right.$ mice) and are reported as means \pm SD. ${ }^{* * *} p<0.001$.

\subsection{Thyroid Cancer Cell Growth Suppression and Apoptosis in BRAF FOXE1 ${ }^{+/-}$Mice}

The different cancer histology was associated with a marked reduction of cell proliferation of $B R A F F O X E 1^{+/}$cancers, as determined by immunohistochemistry (IHC) for Ki-67, compared to BRAF FOXE1 ${ }^{+/+}$(Figure 3A,B). Given the established role of FOXE1 in the survival of developing thyroid follicular cells [16], we also measured apoptosis in thyroid cancers by cleaved caspase-3 staining. As shown in Figure 3C,D, BRAF FOXE1 $1^{+/-}$ cancers show increased apoptosis, as measured by cleaved caspase-3 positive cells number. Both these markers are not detectable in WT and FOXE1 ${ }^{+/+}$treated thyroid (Figure S3).

A

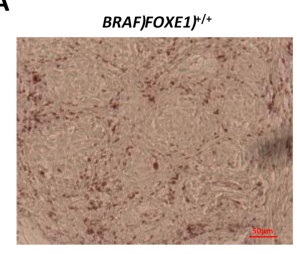

C

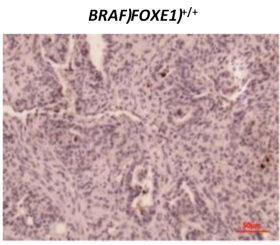

BRAFFOXE1\%

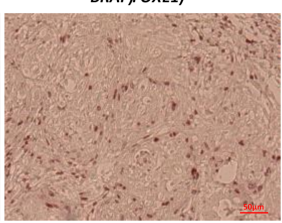

BRAFFOXE1 $)^{\text {r }}$

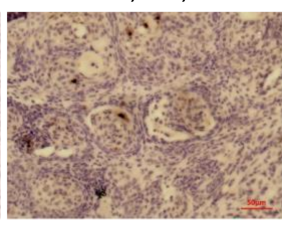

B
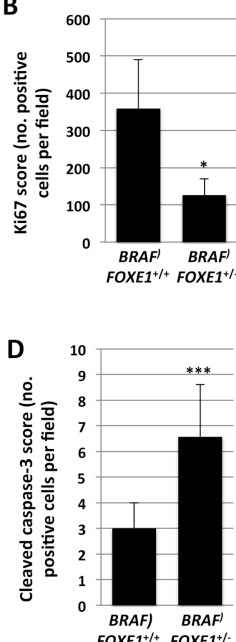

Figure 3. $F O X E 1^{+/-}$thyroid cancer shows reduced proliferation and increased apoptosis. BRAF $F O X E 1^{+/+}$and BRAF FOXE1 $1^{+/-}$mice were treated with doxycycline for one week to induce thyroid cancer. (A). IHC staining for Ki67 was performed on $7 \mu \mathrm{m}$ sections. Here, $20 \times$ magnifications are shown. Each image is representative of six different images. (B). Ki67 positive cells were manually counted in four fields per mouse ( $n=6$ mice). Means of positive cells per field \pm SD are reported. (C). IHC staining for Cleaved Caspase- 3 was performed on $7 \mu \mathrm{m}$ sections. Magnifications of $20 \times$ are shown. Each image is representative of six different images. (D). Cleaved Caspase-3 positive cells were manually counted in four fields per mouse ( $n=6$ mice). Means of positive cells per field \pm SD are reported. ${ }^{*} p<0.05,{ }^{* * *} p<0.001$. 


\subsection{Differentiated Thyroid Gene Expression in BRAF FOXE1 ${ }^{+-}$Cancers}

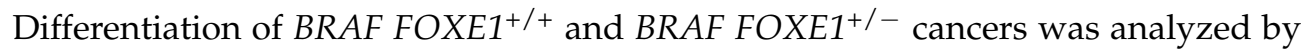
IHC for two representative differentiated thyroid markers, the transcription factor PAX8, and the precursor of thyroid hormones thyroglobulin (TG). IHC analysis demonstrates that the solid structures observed by H\&E staining in FOXE1 ${ }^{+/+}$thyroid cancers are composed of PAX8- and TG-positive cell nests distributed throughout the gland (Figure 4A,B left panels). Conversely, $\mathrm{FOXE1}^{+/-}$cancers show a weaker staining for both PAX8 and TG, with the positive cells surrounding and partially filling the follicle-like structures (Figure $4 \mathrm{~A}, \mathrm{~B}$ right panels). Furthermore, TG staining revealed that the differentiated cells retain thyroglobulin in the cytoplasm in both $F O X E 1^{+/+}$and $F O X E 1^{+/-}$cancers. Indeed, the follicle-like structures observed in $F O X E 1^{+/-}$samples appear to be empty, thus indicating that TG is not released in the lumen. As a control, we analyzed the functional normal WT and FOXE1 ${ }^{+/-}$thyroids, after doxycycline treatment. In both WT and $F O X E 1^{+/-}$follicular cells are PAX8-positive and TG-negative, with the follicle lumens containing TG-positive colloid (Figure S4A).

To further characterize the differentiation state of $B R A F F O X E 1^{+/+}$and $B R A F F O X E 1^{+/-}$ cancers, we analyzed the expression of a panel of thyroid differentiation markers by quantitative RT-PCR. The analysis revealed that in BRAF FOXE1 $1^{+/}$cancers, the decrease of all the analyzed thyroid differentiation markers is significantly more pronounced than that observed in BRAF FOXE1 $1^{+/+}$ones (Figure 4C), showing that lower FOXE1 levels are associated with less differentiated tumors. Conversely, the same analysis performed in control WT and FOXE1 $1^{+/-}$mice confirms that the overall differentiation of $F O X E 1^{+/-}$ thyroids is not reduced compared to the WT ones (Figure S4B).
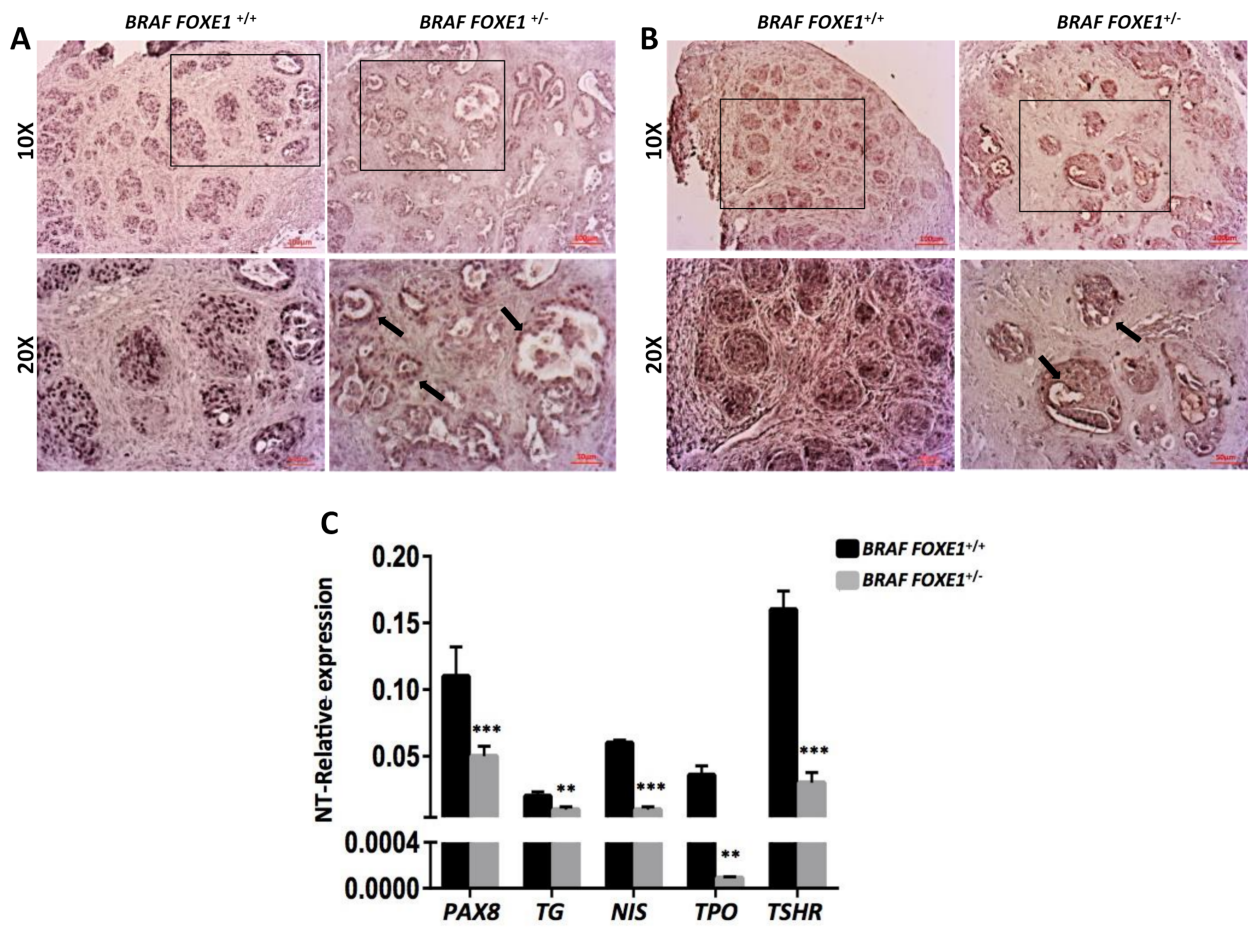

Figure 4. $F O X E 1^{+/-}$thyroid cancer shows decreased expression of differentiation markers. IHC staining was performed on

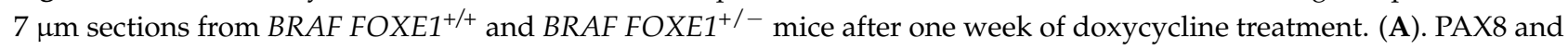
(B). TG. 10X (upper panels) and 20X of the boxed area (lower panels) magnifications are shown. Arrows indicate residual follicular-like structures. Each image is representative of six different images. (C). A panel of differentiation markers was analyzed by quantitative RT-PCR on total RNA extracted from pools of six thyroids form each experimental group: $B R A F F O X E 1^{+/+}$and BRAF FOXE1 $1^{+/-}$mice, either Dox-treated or untreated. Data are normalized by actin expression and reported as the fold change $\left(2^{\wedge}-\Delta \Delta C_{t}\right)$ of treated mice with respect to untreated mice with the same genotype. Means \pm SD are shown. ${ }^{* *} p<0.01,{ }^{* * *} p<0.001$. 


\section{Discussion}

The transcription factor FOXE1 was originally identified as a key player in thyroid development and function, being required since the early stages of thyroid organogenesis for migration and survival of thyrocyte precursors and in the adult thyroid for differentiation maintenance [16,17]. More recently, genome-wide association studies have highlighted a novel role of FOXE1 as a susceptibility gene for thyroid cancer by identifying several non-coding SNPs linked to increased risk of developing PTC [5-7]. Interestingly, most of the identified SNPs fall into putative regulatory regions of the FOXE1 locus, suggesting that different susceptibilities to thyroid cancer could be determined by variable expression level of FOXE1 $[8,9,12]$. This hypothesis is strongly supported by large datasets available online, in which it is reported that FOXE1 expression is higher in normal thyroid with respect to cancer (http:/ / gepia.cancer-pku.cn/detail.php?gene=\&clicktag=boxplot) and that higher FOXE1 expression correlates with longer overall survival (https://www.proteinatlas.org/ ENSG00000178919-FOXE1/pathology/thyroid+cancer).

To establish if different amounts of FOXE1 could result in different outcomes in thyroid tumorigenesis, we generated a mouse model of thyroid cancer harboring only one functional FOXE1 allele by crossing the heterozygous FOXE1 knockout mouse line [16] with a well-established model of thyroid cancer, in which a $B R A F^{\mathrm{V} 600 \mathrm{E}}$ oncogene is expressed in a thyroid-specific and Dox-inducible manner [15]. In the latter, thyroid tumors develop with high penetrance in both thyroid lobes within a week of Dox induction, showing histological and molecular features that closely resemble human high-grade PTC. Here, we show that decreased FOXE1 levels exert pleiotropic effects on thyroid cancer phenotype in vivo. Histological analyses show that reduction of FOXE1 dosage results in the loss of the expected solid pattern of tumor growth [15], with the presence of empty structures resembling follicle remnants, partially filled with cells expressing thyroid markers. The different histology is accompanied by decreased proliferation and increased apoptosis indexes. Interestingly, a similar pattern was observed in the original BRAF mouse model during the recovery of normal thyroid morphology after Dox withdrawal [15]. One possible explanation for this similarity is that FOXE1 reduction in the normal thyroid could hamper the oncogenic process elicited by oncogene activation, likely by decreasing the ability of thyroid cells to survive during transformation. Indeed, the reduced proliferation index and increased apoptosis suggest that $F O X E 1^{+/-}$cancers display a less malignant phenotype.

Besides the morphological differences, we highlighted that reduced FOXE1 function induces a more pronounced loss of follicular cell differentiation, a feature commonly associated with more aggressive thyroid cancers. Such dual oncogenic-anti oncogenic activity of FOXE1 has been recently highlighted by a paper showing that in human thyroid cancers, FOXE1 expression positively correlates with differentiation degree, but also that FOXE1 is able to induce in vitro cell migration and epithelial-to-mesenchymal transition [14] According to these evidences, our in vivo model shows that lower FOXE1 expression leads to the development of less differentiated cancers, which display lower proliferation and higher apoptosis rates, thereby strengthening the possibility that FOXE1 could exert contrasting roles in thyroid neoplastic transformation. Based on these evidences, we might speculate that FOXE1 could act as a lineage-specific oncogene [18-21], meaning that it governs lineage proliferation and survival during development as well as promoting oncogenic phenotypes in cells of the same lineage during carcinogenesis.

In conclusion, our data show for the first time in vivo a cause-effect relationship between FOXE1 loss of function and thyroid cancer features, thus supporting the hypothesis that differential expression of the transcription factor could be the key determinant underlying its role as a susceptibility gene for differentiated thyroid cancer.

\section{Materials and Methods}

\subsection{Mice}

TetO-BRAF ${ }^{\mathrm{V} 600 \mathrm{E}}$ and Tg-rtTA transgenic mice were obtained from Prof. J.A. Fagin at Memorial Sloan-Kettering Cancer Center (New York, NY, USA), where they were generated 
and were crossed to obtain the double transgenic mice $\mathrm{Tg}$-rtTA; TetO-BRAF ${ }^{\mathrm{V} 600 \mathrm{E}}$. The mice were maintained under pathogen-free conditions and controlled temperature, humidity, and light and were supplied with standard or implemented food and water ad libitum in the Italian Ministry-approved (D.M. 78/213-A, and 12/2018-UT) Animal Facility of the University Federico II (Naples, Italy). Tg-rtTA-TetO-BRAF ${ }^{\mathrm{V} 600 \mathrm{E}}$ were crossed with FOXE1 heterozygous mice to obtain FOXE1 wt and FOXE1 heterozygous transgenic mice, required for the study, which were fed with a $2500 \mathrm{mg} / \mathrm{kg}$ doxycycline supplemented fodder for one week. The present study was approved by the Institutional Animal Care and Use Committee (IACUC) of University of Naples Federico II and by the Italian Ministry of Health with protocol no. 2013/0078506.

\subsection{Genotyping}

Genomic DNA (gDNA) was extracted and isolated from mouse tails according to the following protocol: $400 \mu \mathrm{g}$ of Proteinase $\mathrm{K}$ was used in $750 \mu \mathrm{L}$ of lysis buffer ( $50 \mathrm{mM}$ Tris$\mathrm{HCl} \mathrm{pH} 8,100 \mathrm{mM}$ EDTA pH8, $100 \mathrm{mM} \mathrm{NaCl}, 1 \%$ SDS) and added to the samples, which were shaken for $2 \mathrm{~h}$ at $60^{\circ} \mathrm{C}, 250 \mu \mathrm{L}$ of $6 \mathrm{M} \mathrm{NaCl}$ was added, $10^{\prime} 16,000 \times g$ at $4{ }^{\circ} \mathrm{C}, 500 \mu \mathrm{L}$ of isopropanol was added to the supernatant, $10^{\prime} 16,000 \times g$ at $4{ }^{\circ} \mathrm{C}$, and the pellet was washed with $500 \mu \mathrm{L}$ of $70 \%$ ethanol and dissolved in TE (10 mM Tris-HCl, $0.1 \mathrm{mM}$ EDTA). Then, 500 ng of gDNA was used for PCR amplification performed with MyTaq ${ }^{\mathrm{TM}}$ Red Mix (BIO-25044, BIOLINE, Memphis, TN, USA) according to manufacturer's specifications.

\subsection{Quantitative Real-Time PCR}

Total RNA was isolated from frozen mouse thyroids using Trizol (Sigma Aldrich, St. Louis, MO, USA) reagent and total cDNA was generated with SensiFAST cDNA Synthesis Kit (BIO-65054, BIOLINE, Memphis, TN, USA), according to manufacturer's specifications. Quantitative Real-Time PCR on total cDNA was performed with Xpert Fast SYBR (Uni) (GE20, Grisp, Porto, Portugal) using gene specific oligos. FOXE1, PAX8, TG, NIS, TSHR, and TPO oligonucleotides are reported in Credendino et al. [22]. Normalization was performed by amplifying $\beta$-actin with specific oligonucleotides (Fw: $5^{\prime}$-ctgaaccctaaggccaaccgtg-3'; Rev: 5'-ggcatacagggacagcacagcc-3').

\subsection{Staining and Immunohistochemistry}

Thyroids were fixed in $4 \%$ PFA, dehydrated, and paraffin embedded, as already described [23]. Then, $7 \mu \mathrm{m}$ sections were obtained, deparaffinized, and rehydrated. The sections were permeabilized with $5^{\prime}$ PBS- $0.2 \%$ triton and washed $2 \times 5^{\prime}$ PBS. For Hematoxylin/eosin staining, the slides were then stained with hematoxylin (Surgipath 3801562E, Leica, Wetzlar, Germany), washed in ethanol 50\%, and stained in eosin (Surgipath 3801602E, Leica, Wetzlar, Germany). For IHC, they underwent unmasking treatment in citrate buffer $(0.01 \mathrm{M} \mathrm{pH} 6) 15^{\prime}$ in the microwave. Endogenous peroxidases were then saturated with methanol and $1.5 \%$ oxygen peroxide and tissues were permeabilized with $5^{\prime}$ PBS- $0.2 \%$ triton, washed $2 \times 5^{\prime}$ PBS, and blocked in blocking solution (5\% Normal goat serum (S-1000, Vector Laboratories, Burlingame, CA, USA), 3\% BSA, $20 \mathrm{mM} \mathrm{MgCl2,} 0.3 \%$ tween20 in PBS) for $1 \mathrm{~h}$ at room temperature. Primary antibodies were used in blocking solution overnight at $4{ }^{\circ} \mathrm{C}$. The following antibodies were used: PAX8 [22], TG (Dako, Santa Clara, CA, USA), Ki67 (Ab16667, Abcam, Cambridge, UK), and Cleaved Caspase-3 (9661S, Cell Signaling). The sections then underwent the following protocol: $5^{\prime}$ PBS- $0.2 \%$ triton, $2 \times 5^{\prime}$ PBS, $1 \mathrm{~h}$ secondary antibody (biotinylated $\alpha$-rabbit IgG $(\mathrm{H}+\mathrm{L})$ Vector Laboratories BA-1000) 1:100 in blocking solution for $1 \mathrm{~h}$ at room temperature, $5^{\prime} \mathrm{PBS}-0.2 \%$ triton, $2 \mathrm{X}^{\prime} \mathrm{PBS}, 30^{\prime} \mathrm{ABC}$ (Vector Laboratories SK-4000) RT, 5' PBS-0.2\% triton, 3× 5' PBS, DAB substrate (SK-4100, Vector Laboratories, Burlingame, CA, USA). After the staining, the samples were then dehydrated and covered with cover glasses using D. P. X. Mountant (liquid) (GRM655, HIMEDIA Laboratories, Mumbai, India). Images were obtained using Axioskop microscope equipped with an Axiocam 105 color digital camera (Zeiss, Oberkochen, Germany). Images were processed using the Axio Vision software. 
Supplementary Materials: Supplementary materials can be found at https:/ /www.mdpi.com/1422 $-0067 / 22 / 1 / 25 / s 1$.

Author Contributions: Conceptualization, G.D.V. and M.D.F.; methodology, S.C.C.; investigation, S.C.C., C.M., G.D., and A.G.; resources, A.G. and L.D.G.; data curation, S.C.C. and E.C.; writingoriginal draft preparation, S.C.C.; writing—review and editing, G.D.V.; supervision, G.D.V., E.A., C.B., and A.B.; funding acquisition, G.D.V.; All authors have read and agreed to the published version of the manuscript.

Funding: This work was supported by the Italian Ministry of University and Research (MIUR) grant PON03PE_00060_7 and by POR Campania FESR 2014-2020 “SATIN” grant, both to GDV.

Acknowledgments: We thank James Fagin for providing the Tg-rtTA/TetO-BRAF ${ }^{\mathrm{V} 600 \mathrm{E}}$ mouse model and Matilde Gallipari for the technical support in animal handling.

Conflicts of Interest: The authors declare no conflict of interest.

\section{References}

1. Cohen, S.M.; Mukerji, R.; Timmermann, B.N.; Samadi, A.K.; Cohen, M.S. A novel combination of withaferin A and sorafenib shows synergistic efficacy against both papillary and anaplastic thyroid cancers. Am. J. Surg. 2012, 204, 895-900; discussion 900-891. [CrossRef] [PubMed]

2. Chrisoulidou, A.; Boudina, M.; Tzemailas, A.; Doumala, E.; Iliadou, P.K.; Patakiouta, F.; Pazaitou-Panayiotou, K. Histological subtype is the most important determinant of survival in metastatic papillary thyroid cancer. Thyroid Res. 2011, 4, 12. [CrossRef] [PubMed]

3. Kondo, T.; Ezzat, S.; Asa, S.L. Pathogenetic mechanisms in thyroid follicular-cell neoplasia. Nat. Reviews. Cancer 2006, 6, 292-306. [CrossRef] [PubMed]

4. Rebaï, M.; Rebaï, A. Molecular genetics of thyroid cancer. Genet. Res. 2016, 98, e7. [CrossRef] [PubMed]

5. Penna-Martinez, M.; Epp, F.; Kahles, H.; Ramos-Lopez, E.; Hinsch, N.; Hansmann, M.L.; Selkinski, I.; Grunwald, F.; Holzer, K.; Bechstein, W.O.; et al. FOXE1 association with differentiated thyroid cancer and its progression. Thyroid Off. J. Am. Thyroid Assoc. 2014, 24, 845-851. [CrossRef] [PubMed]

6. Wang, Y.; He, H.; Li, W.; Phay, J.; Shen, R.; Yu, L.; Hancioglu, B.; de la Chapelle, A. MYH9 binds to lncRNA gene PTCSC2 and regulates FOXE1 in the 9q22 thyroid cancer risk locus. Proc. Natl. Acad. Sci. USA 2017, 114, 474-479. [CrossRef]

7. Gudmundsson, J.; Sulem, P.; Gudbjartsson, D.F.; Jonasson, J.G.; Sigurdsson, A.; Bergthorsson, J.T.; He, H.; Blondal, T.; Geller, F.; Jakobsdottir, M.; et al. Common variants on 9q22.33 and 14q13.3 predispose to thyroid cancer in European populations. Nat. Genet. 2009, 41, 460-464. [CrossRef]

8. He, H.; Li, W.; Liyanarachchi, S.; Srinivas, M.; Wang, Y.; Akagi, K.; Wang, Y.; Wu, D.; Wang, Q.; Jin, V.; et al. Multiple functional variants in long-range enhancer elements contribute to the risk of SNP rs965513 in thyroid cancer. Proc. Natl. Acad. Sci. USA 2015, 112, 6128-6133. [CrossRef]

9. Tomaz, R.A.; Sousa, I.; Silva, J.G.; Santos, C.; Teixeira, M.R.; Leite, V.; Cavaco, B.M. FOXE1 polymorphisms are associated with familial and sporadic nonmedullary thyroid cancer susceptibility. Clin. Endocrinol. 2012, 77, 926-933. [CrossRef]

10. Somuncu, E.; Karatas, A.; Ferahman, S.; Saygili, N.; Yilmaz, E.; Ozturk, O.; Kapan, M. The investigation of foxe1 variations in papillary thyroid carcinoma. Int. J. Clin. Exp. Pathol. 2015, 8, 13458-13464.

11. Takahashi, M.; Saenko, V.A.; Rogounovitch, T.I.; Kawaguchi, T.; Drozd, V.M.; Takigawa-Imamura, H.; Akulevich, N.M.; Ratanajaraya, C.; Mitsutake, N.; Takamura, N.; et al. The FOXE1 locus is a major genetic determinant for radiation-related thyroid carcinoma in Chernobyl. Hum. Mol. Genet. 2010, 19, 2516-2523. [CrossRef] [PubMed]

12. Landa, I.; Ruiz-Llorente, S.; Montero-Conde, C.; Inglada-Perez, L.; Schiavi, F.; Leskela, S.; Pita, G.; Milne, R.; Maravall, J.; Ramos, I.; et al. The variant rs1867277 in FOXE1 gene confers thyroid cancer susceptibility through the recruitment of USF1/USF2 transcription factors. Plos Genet. 2009, 5, e1000637. [CrossRef]

13. Fan, Y.; Ding, Z.; Yang, Z.; Deng, X.; Kang, J.; Wu, B.; Zheng, Q. Expression and clinical significance of FOXE1 in papillary thyroid carcinoma. Mol. Med. Rep. 2013, 8, 123-127. [CrossRef] [PubMed]

14. Morillo-Bernal, J.; Fernández, L.P.; Santisteban, P. FOXE1 regulates migration and invasion in thyroid cancer cells and targets ZEB1. Endocr. Relat. Cancer 2020, 27, 137-151. [CrossRef] [PubMed]

15. Chakravarty, D.; Santos, E.; Ryder, M.; Knauf, J.A.; Liao, X.H.; West, B.L.; Bollag, G.; Kolesnick, R.; Thin, T.H.; Rosen, N.; et al. Small-molecule MAPK inhibitors restore radioiodine incorporation in mouse thyroid cancers with conditional BRAF activation. J. Clin. Investig. 2011, 121, 4700-4711. [CrossRef]

16. De Felice, M.; Ovitt, C.; Biffali, E.; Rodriguez-Mallon, A.; Arra, C.; Anastassiadis, K.; Macchia, P.E.; Mattei, M.G.; Mariano, A.; Scholer, H.; et al. A mouse model for hereditary thyroid dysgenesis and cleft palate. Nat. Genet. 1998, 19, 395-398. [CrossRef]

17. Fernández, L.P.; López, M.A.; Santisteban, P. Thyroid transcription factors in development, differentiation and disease. Nat. Rev. Endocrinol. 2015, 11, 29-42. [CrossRef] 
18. Bass, A.J.; Watanabe, H.; Mermel, C.H.; Yu, S.; Perner, S.; Verhaak, R.G.; Kim, S.Y.; Wardwell, L.; Tamayo, P.; Gat-Viks, I.; et al. SOX2 is an amplified lineage-survival oncogene in lung and esophageal squamous cell carcinomas. Nat. Genet. 2009, 41, 1238-1242. [CrossRef]

19. Kwei, K.A.; Kim, Y.H.; Girard, L.; Kao, J.; Pacyna-Gengelbach, M.; Salari, K.; Lee, J.; Choi, Y.L.; Sato, M.; Wang, P.; et al. Genomic profiling identifies TITF1 as a lineage-specific oncogene amplified in lung cancer. Oncogene 2008, 27, 3635-3640. [CrossRef]

20. Pan, J.; Silva, T.C.; Gull, N.; Yang, Q.; Plummer, J.T.; Chen, S.; Daigo, K.; Hamakubo, T.; Gery, S.; Ding, L.W.; et al. Lineage-Specific Epigenomic and Genomic Activation of Oncogene HNF4A Promotes Gastrointestinal Adenocarcinomas. Cancer Res. 2020, 80, 2722-2736. [CrossRef]

21. Yamaguchi, N.; Ito, E.; Azuma, S.; Honma, R.; Yanagisawa, Y.; Nishikawa, A.; Kawamura, M.; Imai, J.; Tatsuta, K.; Inoue, J.; et al. FoxA1 as a lineage-specific oncogene in luminal type breast cancer. Biochem. Biophys. Res. Commun. 2008, 365, 711-717. [CrossRef] [PubMed]

22. Credendino, S.C.; Bellone, M.L.; Lewin, N.; Amendola, E.; Sanges, R.; Basu, S.; Sepe, R.; Decaussin-Petrucci, M.; Tinto, N.; Fusco, A.; et al. A ceRNA Circuitry Involving the Long Noncoding RNA Klhl14-AS, Pax8, and Bcl2 Drives Thyroid Carcinogenesis. Cancer Res. 2019, 79, 5746-5757. [CrossRef] [PubMed]

23. Credendino, S.C.; Lewin, N.; de Oliveira, M.; Basu, S.; D'Andrea, B.; Amendola, E.; Di Guida, L.; Nardone, A.; Sanges, R.; De Felice, M.; et al. Tissue- and Cell Type-Specific Expression of the Long Noncoding RNA Klhl14-AS in Mouse. Int. J. Genom. 2017, 2017, 9769171. [CrossRef] [PubMed] 